\title{
Concepts and Constructs of Urban Sensing
}

Scott W. Cunningham

Faculty of Technology, Policy and

Management

Delft University of Technology

2600 GA, Delft, The Netherlands

s.cunningham@tudelft.nl

\author{
Alexander Verbraeck \\ Faculty of Technology, Policy and \\ Management \\ Delft University of Technology \\ 2600 GA, Delft, The Netherlands \\ s.cunningham@tudelft.nl
}

\begin{abstract}
There is an increasing practice of combining various sorts of data from the city to obtain a comprehensive operating picture for urban managers. The sorts of data being combined includes administrative data, sensor and transactional data, and social media data. In order for these practices to fulfil their full promises it is important that practitioners build systems which measure the correct things. It is also important that theorists develop systematic explanations for complex urban phenomena. This paper confronts the challenges of building better concepts and constructs for urban data analysis and sensing.
\end{abstract}

Keywords - Urban science, big data, internet of things, sensors, data governance, information systems, administrative data

\section{INTRODUCTION}

The purpose of this paper is to argue that urban data collection requires improved theoretical concepts to better guide and structure data collection. In order to further pursue this question it is necessary to survey the range of technologies available for collecting data about the structure and functioning of the modern city. It is important to also consider the challenges attendant upon the collection of this data. The data must be clean, standardized, and appropriately processed.

Urban data is actively collected for multiple potential purposes. These purposes rate from reports or summaries of urban conditions, to more complex policy and decisionmaking tasks. Developing plans for intervention is one such task. An even greater challenge is to consider counterfactuals - the range of possible policy outcomes, considering the costs, benefits and consequences of action or inaction [1]. By evaluating sources of data we learn about the urban environment, but also equally about the political intents and purposes of the policy makers which commission the work [2].

Given this dual process of creation and discovery it is important to consider those concepts which are the most valid and valuable for urban sensing. By consideration of such processes this paper helps to add rigor to the collection and compilation of urban data. Such consideration further enables decision-makers to better clarify the ranges of problems that can or cannot be solved through the appropriate collection and use of data [3]. Given the importance of theories in data collection and analysis it is important to formulate and select the most appropriate concepts for use in support of decision-making. As a result it is helpful to use frameworks of concept validity to evaluate the suitability of a range of emerging concepts of cities and their data [4].
Despite the increasing prevalence of the smart city concept, and attendant technologies such as the industrial internet, or the internet of things, there has been little attention paid in the literature to data collection processes in the city. Much of the extant literature instead addresses individual technologies, or potential applications of urban data. Despite these efforts there has been little attention paid in the literature to the purposes for which this data is being collected, and the range of valid possible inferences which could be drawn from such data. There are notable exceptions. There is a notable literature on the sociological and political analysis of big data [2]. This is a useful adjunct to a broader literature on the sociology and philosophy of science. Another source provides an ever expanding list of possible applications of data analysis in the city [3]. And still other sources have productively discussed a vision of urban data including architectural elements, scenarios and visions, and potential areas for future research [5]. This paper therefore contributes to an important gap in the literature addressing the systematic evaluation of data collection efforts at the city level.

Consequently the paper is organized by examining the instruments and sources of data which are collected in pursuit of a common operating picture of the city (section 2). This includes a survey of available instruments for urban sensing (section 2.1), and a consideration of the challenges that such data present for standardization and processing (section 2.2). This needs to be assembled into thematic maps or other constructs, given an understanding of the complexity of urban processes. This is review in section 3 . The validity of the available concepts are examined in section 4 . The paper concludes with recommendations and prospects for future work in the field of smart cities, urban sensing, and data analysis. Given this evaluation of available concepts in section 5, this paper may help to enrich the theoretical basis of an emerging field of urban science and analytics. Developing a common operating Picture of the City

\section{DEVELOPING A COMMON OPERATING PICTURE OF THE CITY}

This section examines the instruments and sources of data collected in pursuit of a common operating picture of the city, for decision-makers and for the operational managers of the city. New technologies provide decisionmakers with an enhanced capability to measure, infer and better understand what is going on in our urban environments [5]. Measurements include patterns of urban mobility, the location and nature of pollution, the intake of materials and energy, and the full extent of the urban environment including land use, impermeable land and 
greenspace. Together this information enables the city to develop a common operating picture, and may increasingly be delivered as information services for consumption by the city and its citizens $[5,6]$.

In section 2.1 a large variety of possible urban sensing technologies are broadly surveyed. In order to concisely present the broad array of relevant technologies it is necessary to structure the material. The section examines the variety of data sources in the city in terms of the instruments which are used. The extent of data collection devices and instruments, the instrumentation platforms used, and the extent of collection are all considered. This review also considers a variety of different urban planning needs for this sensing data. The efforts required to impose rigor and standardization on the data for decision-making is considered in section 2.2. Part of the challenge lies in creating common definitions and operating standards. A substantial portion of the challenge also lies in sampling, fusing or filtering a variety of different sources of information to create a complete picture of the city.

A framework which could map the various sensor inputs and outputs could be useful. For instance, it is possible to measure the passage of road traffic through fiber optic cables. These cables convert weight into stress, and this stress results in changes to the optics of the fiber. This enables even very subtle measurements of the speed, volume and frequency of traffic to be made. Unfortunately a comprehensive picture of the design opportunities for urban platforms does not yet seem to exist. There are however related works from an engineering science and engineering design perspective [7].

This perspective is very compatible with sensor technologies measure inputs, and deliver responses via actuators. The biggest differences between the design framework, and any comprehensive sensor framework lies with transponders. Transponders convert sensor input into a variety of potential signals for use by actuators. Smart city technologies are advancing very rapidly because of the increasing presence of digital transponders powered by electronic devices and machine learning technologies. These transponders impute measurements based on previous laboratory or ground truth measurements. For instance particulate matter in the air has significant negative health effects on citizens. Unfortunately it is difficult to make comprehensive measurements across the whole of an urban space. As a consequence ground truth measurements are made, for instance in a laboratory setting, on the effects of particulate matter on the spectrum of visible daylight. These measurements can then be used to impute actual particulate levels across the city using comparatively cheap and portable equipment such as a smart phone. Needless to say though, the switch from actual measurements to the imputation of measurement is a significant change worthy of appropriate monitoring and governance.

\section{A. Available Instruments for Sensing}

One can best characterize the variety of urban sensing technologies and approaches by considering the instrumentation used, the platforms used which contain instrumentation, the applications of the sensing technology, and the institutional modes used for data collection. There are a wide set of possible channels of data to be collected in pursuit of a common operating picture of the city. These channels can be broadly characterized as text, numerical, electromagnetic, biochemical, and mechanical. In the following paragraphs each of these channels is discussed in turn.

There is the potential to collect text data, for instance from social media or city ordinances. Traditional census data provides numerical indices of numbers of citizens, and numbers of businesses. Cell phone data provides additional relational context, for instance about the mobility of citizens across the city. Economic surveys extend this information with numerical indicators of trade, finance, production and income. Beyond these measures there are a wide range of electromagnetic sensors available, which register information at the $\mathrm{x}$-ray, optical and radio bands. Instruments providing multispectral read-outs on imagery are also available. Biochemical sensors detect individual chemicals, or the presence of particular forms of biological life. For instance biosensors are used to detect the presence of viruses in untreated waste water. A range of mechanical sensors are also available. The ability to register the noise environments is of critical importance. Other relevant sensor techniques establish vibration, or the presence of stress fractures in buildings or solid materials.

A further distinction occurs according to the scale, or layer of measurement that automated collection is occurring. For the smallest of scales this entails the use of RFID tags, and other embedded sensors and actuators. These are built up into devices with software applications, such as a smart phone. There are also expensive and high-end sorts of equipment such as x-ray devices and electron microscopes. Networked applications serve as an intermediate layer which conveys data, but also serves as an important indicator of population-wide activities. Networks also permit new governance models of participation and crowd sensing. Weather stations also fit into this category of networked instrumentation. Finally there are large-scale and remote applications such as satellite imagery.

The scale of data collection was discussed previously, but the platforms for the collection of data are also highly varied. One can characterize manual and automated procedures for data collection. One can also characterize the data according to whether it has been volunteered, or passively collected. Finally we could characterize the collection according to whether formal or informal institutions for data collection have been employed. For instanced in crowd sensing applications, individual citizens work together in temporary and shifting collectives to continuously monitor the city.

Applications for city sensing are extremely varied. Broadly speaking we can divide applications into planning and governance applications, environmental services, and asset management applications. In the governance domain there are applications for population counting, health care, hazard monitoring, as well as applications in regulation, such as verification, compliance, and enforcement. Environmental services include applications for sustainability, characterization of green space, air quality measurement, land use planning and ground cover, bioassays, and waste remediation and recycling. Asset 
management includes applications in transportation systems, building recognition, and infrastructure appraisal [8-14]

\section{B. Challenges for Data Standardization and Processing}

Such measurements are not without difficulties, regardless of the quality of the instrumentation or the platform. Previous work has examined the challenges involved in collecting remote sensing data. To a large extent the same concerns apply to the varieties of data collected in urban and city sensing as well. For example the data which is being collected is fundamentally noisy and uncertain. As a result these measures should be subjected to a process of data compilation and assessment. This entails the design of protocols for sampling the data. Such procedures should be efficient, rigorous, and easy to implement $[15,16]$. Once the data is compiled, additional requirements are needed to assure accuracy in comparison and reporting (Foody 2002). But even then such rigorous testing may not be enough to ensure the valid production of data.

Furthermore it is abundantly clear that the data which is being collected must be better standardized. Standardization enables correct comparisons to be made across time and across urban environments [16]. Recent ISO standards have been developed to ensure a common set of measures defining the performance of city services, and the quality of urban life. The objectives of this measure are to allow comparisons between cities and regions regardless of their respective sizes [17]. Standards such as these encourage collection of data across multiple thematic areas including education, shelter, health, transportation and innovation [18].

These challenges for effective data collection raise multiple possible issues for validity. One is whether the data collected actually measures what it purports to measure; this is measurement validity. Another issue is whether the data collected measures all potential facets of an underlying issue; this is content validity. A final issue is whether there is a principled and systematic process to organize measurements. This aspect involves the creation of measurement constructs [4]. This diverse range of data, platforms and applications demands the appropriate use of concepts for the appropriate collation of urban data sources. This is the topic of the following section.

\section{COMPLEX URBAN PROCESSES}

Measurement cannot be effectively made without the use of theory. Isolated measures are combined to produce thematic maps over time and space. The city itself emerges out of a complex and dynamical process of interactions between people, places and the available infrastructure. These processes generate the city, but also generate the data which can be used in tracking the growth, health and evolution of the city [1]. When considering urban growth, assessments of population and economic product are insufficient to gain a full picture of the underlying dynamics. There are a variety of factors underlying growth. Some of the factors such as landscape conditions, infrastructure availability and transport costs, require the construction of detailed inventories before an assessment can be made [19]. Furthermore there is still more to be understood concerning a city than merely the growth of its physical infrastructure. Other factors such as policies, institutions, and the formal and informal economy may not be able to be directly observed at all [20].
The following brief review of complex urban processes therefore breaks the literature down into two parts. The first concerns the growth of the city within an environmental context. This addresses the citing of the city and its expansion. The expansion of a city can be considered over time, in area, and with regard to the growth of a supporting network of roads and other infrastructure. The second topic addresses the social context of the city, the observable factors such as policies, institutions and the economy that may be difficult to directly measure using available data. This topic also addresses the complex relationships formed between the citizens of the city, and amenities including infrastructure, employment, and a clean environment.

\section{A. Patterns of Urban Growth}

The citing of a city is extremely significant to future prospects for growth. City citing only partially corresponds to the presence of a large population base, or the availability of arable land [21]. Nonetheless there are complex relationships between climate, economic growth and urbanization [22]. Regardless once cited, the presence of a city may be remarkably long-lived [23, 24]. It's unclear whether the persistence of urban growth is because of a relatively limited selection of favorable citing, or the longterm economic advantages created by public goods provision.

The growth of a city can be measured over time, as well as over space. This discussion begins by considering the temporal patterns of urban growth. The growth dynamic of a city is strongly dependent on its population, which then is affected by its economic growth regime. Cities dependent upon physical infrastructure grow asymptotically to match the available resources. Cities dependent on social goods, such as art, culture or innovation grow hyperbolically, rapidly outstripping the resources devoted to their growth. The city must then undergo repeated restructuring or rationalization events in order to maintain its growth. An administrative and planning challenge lies in creating flexible options for the sustained growth of the city. Policies of over-investment may then result in long-term declines in urban quality which are difficult to reverse [25].

The only other option for a city which has outstripped its resource base is diminishment and decay [26]. The almost biological character of cities and growth has led many authors to consider metabolism as a ready model of how cities transform raw material and energy into the goods and services which are readily favored by humans. The concept of metabolism also invites planners and decision-makers to consider the sustainability, efficiency and resilience of the urban environment [27].

Cities can also be measured by their spatial extent. When measured over space the growth of a city can be measured by total area of different kinds of land use or habitation density. Cities demonstrate a complex pattern of growth at their cores as well as at their peripheries [28]. Alternatively more lineal measures of infrastructure might be used, counting for instance the total kilometers of transport, water, electricity, or telecommunications lines which feed the city. In a lineal explanations of growth major infrastructures arteries become a corridors for further growth. 


\section{B. Socio-Economic Complexity of the City}

Any account of the city which neglects the interaction of citizens with the built infrastructure would of course be fundamentally complete. This brief review ties together various literatures which discuss how citizens interact with the built environment, how governance and public goods helps determine urban functioning, and how cities foster innovation and economic production. The review begins with the complex manner in which cities match scarce resources and amenities to their citizens.

The matching of goods and services to consumers or citizens invites both economic as well as political considerations. From an economic perspective the economic functions of the city are governed by markets. Market mechanisms help to coordinate the allocation of land to those with both a desire and a willingness to pay. Cities host a variety of different institutions, ranging from multinational firms, smaller local firms, and local or national government offices. The balance of these institutions creates enduring opportunities for economic growth [29].

Despite the economic provisioning of the city, many key features of the city extend far beyond the marketplace. Governments make decisions about the provision of public goods, the balance of public and private land, and the overall zoning of a city. A full accounting of urban life therefore also requires a political consideration of cities. One political account is of growth advocacy, where elite coalitions steer urban decision-making towards the choices which are most favorable to their own individual interests [30]. Protest movements, for instance among a public dispossessed of affordable housing, is also an important process of political and collective action often occurring within urban areas [31].

There is a complex relationship between pollution and economic development. Initial economic growth and development generally results in a generally worsening pattern of exposure to a variety of pollutants. However beyond a certain amount of economic development, and this amount varies by pollution source, there is generally improving prognosis for environmental quality [32]. This account of urban pollution suggests that exposure to pollution is evenly distributed throughout the population, when pollution is in fact quite unevenly distributed even in the most developed of cities. For instance exposure to even routine sources of air pollution is increasingly implicated in a range of negative health outcomes [33]. Furthermore vulnerable populations may be disproportionately exposed to such pollutants [34].

Large cities support a vibrant urban geography. Large cities display a greater variety of professions. The agglomeration of skilled trades and knowledge in turn results in a higher domestic product, and higher levels of innovation. Larger cities further sustain a higher diversity of leisure activities, and thereby enable a greater production and consumption of both artistic and cultural activities [26, 35, 36]. Given the challenges of attuning resource consumption in cities, a particular need of urban management is appraising levels of urban complexity.

This review demonstrated a number of different conceptual accounts of the city, ranging from physical and infrastructural perspectives, to natural resource perspectives, to the political economy of the city. This review has not yet discussed how these accounts of the city can be deployed to create a comprehensive operating picture of the city. One prominent source characterizes a range of urban problems using a typology of infrastructure problems, health problems, regulatory problems, public safety problems and problems of human services provision. It is important to inquire whether there is an effective match between the theoretical accounts of the city, and the practical problems facing public administrators. Furthermore if there is a short-coming it is important to ask whether there is a lack of available theory, or whether there is a lack of public imagination in applying available knowledge to the solution of these problems.

\section{VALID CONCEPTS FOR URBAN SENSEING}

The gathering of data and the creation of theories about the city is driving a number of new goals, challenges, and smart city projects. Researchers are calling for a new understanding of social problems in light of data, and confronting new challenges in understanding the city as a laboratory for innovation. Future scenarios of urban life anticipate entirely new intelligence functions, modes of governance, and planning structures driven from the collection of new data). This section provides an opportunity to evaluate the urban concepts presented in the previous section. This evaluation is two-fold. The cross-comparison between theory and data reveals both theoretical as well as practical opportunities for urban sensing. Furthermore this section evaluates the concepts most likely to lead to productive and valid inferences concerning urban growth and resilience.

\section{A. Validity}

The first half of this paper described technological capabilities to measure the city, creating new capabilities to create a common operating picture of the city. Increasingly this data collection will need to be better structured. Such structuring enables better synthesis and standardization of data, and a closer approach to the needs of policy makers. Information and data will not be effectively leveraged without new theories of urban functioning.

In this light a range of different conceptual pictures of the city are presented. However not all theories are made equal. Which theories should we select given the technological capabilities of urban sensing, the practical concerns of modern urban design and management, and the realization that statistical collection is a political and an administrative practice? A primary objective of science is to deliver "simple, systematically unified account of empirical phenomena [37]."

This section therefore considers which concepts and constructs of urban analysis are likely to be the most valid, and thereby create the most accurate and effective portraits of the urban environment for urban planners and policy makers. There are a broad range of existing criteria designed for help in selecting effective concepts. These operational tests of theories are derived from practices of case study research and of quasi-natural experimentation. A common set of tests applied to theoretical concepts include the following:

- Observational validity: Is the concept reducible to observations? 
- Criterion related validity: Does the concept enable theorists to predict the value of other concepts of theoretical or practical interest?

- Systemic validity: Does the concept enable the integration of previously disparate concepts, or the creation of whole new conceptual systems?

- Semantic validity: Are the terms and definitions used by the concept clear, and do they enable consistent usage?

- Control validity: Does the concept enable influence and control over a system of interest? [4]

Given this it is important to note that cities are difficult entities to govern and control. The literature often speaks of the organic or metabolic character of cities, as if the urban environment operates partly under its own rules and principles somewhat removed from the sphere of human action and decision-making. This makes control validity a difficult test for any theory of urban functioning and data collection. Another challenge lies in the whole space of semantic validity. Despite efforts to create a grammar of city components, there is still only a limited common vocabulary available for describing urban phenomena across time and across place.

That said some available theories do seem able to address the multiple criteria set forth above, for the space of urban analysis and design. Arguably theories of urban growth and morphology have a number of desirable features for further research. The study of urban form is highly conducive to empirical measurement, especially given a new realm of technological opportunities raised by urban analysis and remote sensing. The study of urban form is also comparatively unchallenged as far as creating a common vocabulary using semantically consistent components.

Another promising avenue for theoretical development lies in the recognition that cities are social reactors, hastening economic development, innovation, and the assemblage of complex skills, products and services. Such theories have a strong criterion related validity - they seem to enable theorists to predict many other concepts of theoretical and practical interest. Furthermore such theories promise strong opportunities for further systematization. Many seemingly disparate concepts might be unified by means of such theories. One promising example of such theories in action is the social reactors project of the Sante Fe Institute.[38].

\section{B. Opportunity}

This review reveals opportunities - both in the novel application of data, as well as in creating relevant new theories of urban function. Theoretically there needs to be better models of urban trust and social cohesion. There are systematic relationships between urban crime, blight and vandalism and the overall quality of the neighborhood. Central to the functioning of the neighborhood is the level of trust displayed in local officials as well as in potential investors who might spearhead neighborhood investment [39]. Such a theory might guide the redevelopment of brownfield zones, and enable more effective cooperation with local communities [40]. Further theoretical opportunities lie in systematic explanations of social contagion. There is a growing body of evidence that neighborhoods shape individual outcomes, although there is little understanding of the causal mechanisms involved. Some of these conceptual links involve socialization by adults, peer influences, social networks, and exposure to crime and violence. Humans are subject to convergent behaviors created by local interaction even in the absence of centralization coordination [41]. Policy makers require more support in determining how neighborhoods matter and for whom [42].

There seems to be new practical opportunities as well. It is clear that there are a broad range of problems of planning, zoning and urban design which could soon be substantially assisted by enhanced regimes of urban data sensing and data collection. Initial developments in this direction are very promising, including the enhanced use of data for green space design and microclimate development in cities [43], and the data-oriented design of neighborhoods to bring residents closer to desired destinations, and to enhance walkability [44]. Judging by the literature these data-oriented design developments are relatively new. There seems considerable opportunity to systematize and expand the practice.

\section{CONCLUSIONS}

This paper provided a high-level overview over many of the component technologies and platforms currently being deployed to create a comprehensive view of the city. These technologies potentially solve some problems, but also introduce new challenges of data integration, design and synthesis. Since these are such new technologies it is not yet fully apparent where the highest opportunities lie for practical deployment. Nor is it fully apparent which of many theories or concepts of urban design and functioning are likely to be most conducive to the collection of vast quantities of new data. Previous research in the sociology of statistics and data has necessarily been retrospective in character [2]. Such work is extremely valuable, but it is also constructive to be forward looking, anticipating new societal needs, and new administrative contexts.

By means of this systematic and problem-oriented analysis of the field, this paper makes two contributions to the literature. First the paper helps to address important gap in the literature by considering the systematic character of current data collection efforts at the city level. Secondly the paper help to enrich the theoretical basis of an emerging field of urban science and analytics by selectively surveying available concepts of urban functioning, and by evaluating these concepts according to principles of experimental validity.

In this concluding part of the paper it is important to acknowledge the limitations of the work. This paper applied principles of conceptual validity to a limited selection of possible theories of cities and their function. The objectives of such an analysis is not to foreclose opportunities for pure research. When evaluating available theories we can help to identify the most promising opportunities for research in this specific context of urban analysis, urban planning, and data sensing. This paper is entirely conceptual in character, and this itself is an important limitation of the work. Furthermore despite this framing of potential areas of research and practical promise is necessarily a subjective exercise. 
It is important to recognize that cities are complex objects, studied from multiple perspectives and for multiple reasons. This review of urban concepts is necessarily very circumscribed, and driven for very specific purposes. Despite this the paper examined a very broad selection of literature ranging from engineering, economics, politics, sociology, urban planning, geography and development. The field of urban geography, analysis and planning remains a strongly interdisciplinary field.

\section{REFERENCES}

[1] Pearl, J., Glymour, M., and Jewell, N.P.: 'Causal Inference in Statistics: A Primer’ (John Wiley \& Sons Ltd., 2016. 2016)

[2] Desrosières, A.: 'The Politics of Large Numbers: A History of Statistical Reasoning' (Harvard University Press, 1998. 1998)

[3] https://datasmart.ash.harvard.edu/news/article/how-can-data-andanalytics-be-used-to-enhance-city-operations-723

[4] Cook, T.D., and Campbell, D.T.: 'Quasi-Experimentation: Design \& Analysis Issues for Field Settings' (Houghton Mifflin, 1979. 1979)

[5] Gubbi, J., Buyya, R., Marusic, C., and Palaniswami, M.: 'Internet of Things (IoT): A vision, architectural elements, and future directions', Future Generation Computer Systems - The International Journal of eScience,, 2013, 29, (7), pp. 1645-1660

[6] Perera, C., Zaslavsky, A., Christen, P., and Georgakopoulos, D.: 'Sensing as a service model for smart cities supported by Internet of Things', Transactions on Emerging Telecommunications Technologies, 2014, 25, (1), pp. 81-93

[7] Dieter, G.E., and Schmidt, L.C.: 'Engineeering Design' (McGrawHill, 2002. 2002)

[8] Batty, M., Axhausen, K.W., Giannotti, F., Pozdnoukhov, A., Bazzani, A., Wachowicz, M., Ouzounis, G., and Portugali, Y.: 'Smart cities of the future', European Physical Journal - Special Topics, 2012, 214, (1), pp. 481-518

[9] Berardino, P., Fornaro, G., Lanari, R., and Sansosti, E.: 'A new algorithm for surface deformation monitoring based on small baseline interferograms', IEEE Transactions on Geoscience and Remote Sensing,, 2002, 40, (11), pp. 2375-2383

[10] Calabrese, F., Colonna, M., Lovisolo, P., Parata, D., and Ratti, C.: 'Real-time urban monitoring using cell phones: A case study in Rome', IEEE Transactions on Intelligent Transportation, 2011, 12, (1), pp. 141-151

[11] Denk, M., Glasser, C., Kurz, T.H., Buckley, S.J., and Drissen, P.: 'Mapping of iron and steelwork by-products using close range hyperspectral imaging: A case study in Thuringia, Germany', European Journal of Remote Sensing, 2015, 48, pp. 489-509

[12] Iqbal, S.S., Mayo, M.W., Bruno, J.G., V., B.B., Batt, C.A., and Chambers, J.P.: 'A review of molecular recognition technologies for detection of biological threat agents', Biosensors and Bioelectronics, 2000, 15, (11-12), pp. 549-578

[13] Khan, W.Z., Xiang, Y., Aalsalem, M.Y., and Arshad, Q.: 'Mobile phone sensing systems: A survey', IEEE Communications Surveys and Tutorials, 2013, 15, (1), pp. 402-427

[14] Rodriguez, M., Sanders, C.A., and Greenbaum, E.: 'Biosensors for rapid monitoring of primary-source drinking water using naturally occurring photosynthesis', Biosensors and Bioelectronics, 2002, 17, (10), pp. 843-849

[15] Stehman, S.V., and Czaplewski, R.L.: 'Design and analysis for thematic map accuracy: Fundamental principles,' Remote Sensing of Environment, 1998, 64, (3), pp. 331-344

[16] Stehman, S.V.: 'Basic probability sampling designs for thematic map accuracy assessment', International Journal of Remote Sensing, 1999, 20, (12), pp. 2423-2441

[17] https://www.iso.org/standard/68498.html, accessed 27 July 2018

[18] Fox, M.S.: 'The role of ontologies in publishing and analyzing city indicators', Computers Environment and Urban Systems, 2015, 54, pp. 266-279

[19] Antrop, M.: 'Landscape change and the urbanization process in Europe', Landscape and Urban Planning, 2004, 67, (104), pp. 9-26

[20] Seto, K.C., Fragkias, M., Guneralp, B., and Reilly, M.K.: 'A metaanalysis of global urban land expansion,' PLOS ONE, 2011, 6, (8), pp. e23777
[21] Zhou, Y., Smith, S.J., Zhao, K., Imhof, M., Thomson, A., BondLamberty, B., Asrar, G.R., Zhang, X., Chunyang, H., and Elvidge, C.D.: 'A global map of urban extent from nightlights', Environmental Research Letters, 2015, 10, pp. 054011

[22] Mellinger, A.D., Sachs, J.D., and Gallup, J.L.: 'Climate, water navigability, and economic development', in Clark, G.L., Feldman, M.P., and Gertle, r.M.S. (Eds.): 'The Oxford Handbook of Economic Geography' (Oxford University Press, 2000)

[23] http://web.econ.ku.dk/pabloselaya/papers/RomanRoads.pdf

[24] Ortman, S.G., Cabaniss, A.H.F., Sturm, J.O., and Bettencourt, L.M.A.: 'The Pre-History of Urban Scaling', PLOS ONE, 2014, 9, (2), pp. e87902

[25] Tainter, J.A.: 'The Collapse of Complex Societies' (Cambridge University Press, 1988. 1988)

[26] Bettancourt, L.M.A., Lobo, J., Helbing, D., Kuhnert, C., and West, G.B.: 'Growth, innovation, scaling, and the pace of life in cities', Proceedings of the National Academy of Sciences, 2007, 104, (17), pp. 7301-7306

[27] Pincetl, S., Bunje, P., and Holmes, T.: 'An expanded urban metabolism method: Toward a systems approach for assessing urban energy processes and causes', Landscape and Urban Planning,, 2012, 107, (3), pp. 193-202

[28] Makse, H.A., Havlin, S., and Stanley, H.E.: 'Modelling urban growth patterns', Nature, 1995, 377, pp. 608-612

[29] Markusen, A.: 'Sticky places in slippery space: A typology of industrial districts', Economic Geography, 1996, 72, (3), pp. 293-313

[30] Molotch, H.: 'The city as a growth machine', American Journal of Sociology, 1976, 82, pp. 309-330

[31] Lipsky, M.: 'Protest as a political resource', American Political Science Review, 1968, 62, pp. 1144-1158

[32] Grossman, G.M., and Krueger, A.B.: 'Economic growth and the environment', Quarterly Journal of Economics, 1995, 110, (2), pp. 353-377

[33] Pope, C.A., Burnett, R.T., Thun, M.J., Calle, E.E., Krewski, D., Ito, K., and Thurston, G.D.: 'Lung cancer, cardiopulmonary mortality, and long-term exposure to fine particulate air pollution', JAMA Journal of the American Medical Association, 2002, 287, (9), pp. $1132-1141$

[34] Gee, G.C., and Payne-Sturges, D.C.: 'Environmental health disparities: A framework integrating psychosocial and environmental concepts', Environmental Health Perspectives, 2004, 112, (17), pp. 1645-1653

[35] Hildago, C.A., and Hausmann, R.: 'The building blocks of economic complexity', Proceedings of the National Academy of Sciences, 2009, 106, (26), pp. 10570-10575

[36] Quercia, D., Aiello, L.M., and Schifanella, R.: 'Diversity of indoor activities and economic development of neighborhoods', PLoS ONE, 2017, 13, (6), pp. e0198441

[37] Hempel, C.G.: 'Philosophy of natural science' (Prentice-Hall, 1966. 1966)

[38] https://www.santafe.edu/research/initiatives/social-reactors-project

[39] Greenberg, M.R.: 'Improving neighborhood quality: A hierarchy of needs', Housing Policy Debate, 1999, 10, (3), pp. 601-624

[40] Greenberg, M. R., and Lewis, M.J.: 'Brownfields redevelopment, preferences and public involvement: A case study of an ethnically mixed neighbourhood', Urban Studies, 2000, 37, (13), pp. 2501-2514

[41] Raafat, R.M., Chater, N., and Frith, C.: 'Herding in humans', Trends in Cognitive Science, 2009, 13, (10), pp. 420-428

[42] Ellen, I.G., and Turner, M.A.: 'Does neighborhood matter? Assessing recent evidence', Housing Policy Debate, 1997, 8, (4), pp. 833-866

[43] Bowler, D.E., Buyung-Ali, L., Knight, T.B., and Pullin, A.S.: 'Urban greening to cool towns and cities: A systematic review of the empirical evidence', Landscape and Urban Planning, 2010, 97, (3), pp. $147-155$

[44] Handy, S., Cao, X.Y., and Mokhtarian, P.: 'Correlation or causality between the built environment and travel behavior? Evidence from Northern California', Transportation Research Part D- Transport and Environmennt, 2005, 10, (6), pp. 427-444 